\title{
Satisfação dos pacientes com doença de Chagas atendidos por um serviço de atenção farmacêutica no estado do Ceará, Brasil
}

\author{
Satisfaction of Chagas disease patients attended \\ at a pharmaceutical care service in the State of Ceará, Brazil
}

\author{
Alanna Carla da Costa ${ }^{1}$ \\ Darlan da Silva Cândido ${ }^{2}$ \\ Arduina Sofia Ortet de Barros Vasconcelos Fidalgo ${ }^{1}$ \\ José Damião da Silva Filho ${ }^{1}$ \\ Carlos Eduardo Menezes Viana ${ }^{1}$ \\ Monise Anne Lima ${ }^{1}$ \\ Mônica Coelho Andrade ${ }^{1}$ \\ Maria de Fátima Oliveira ${ }^{1}$
}

1 Universidade Federal do Ceará. R. Capitão Francisco Pedro 1210, Rodolfo Teófilo. 60430-372 Fortaleza CE Brasil. alannacarla.costa@ gmail.com

${ }^{2}$ Instituto do Coração, Universidade de São Paulo. São Paulo SP Brasil.
Abstract In 2005, a pharmaceutical care service was created in the State of Ceará to provide pharmacotherapeutic follow-up for individuals infected with Trypanosoma cruzi (Chagas Disease). After 10 years of operation, an evaluation was conducted to assess the degree of satisfaction of patients treated under the service. This prospective study used a questionnaire comprising the following sections: socioeconomic data; infrastructure, facilities and operations; pharmaceutical care; and importance of the service. Seventy patients of both sexes and over 18 years of age were interviewed between August 2014 and May 2015. As for infrastructure, location and operation, the average grades show a high level of patient satisfaction. Regarding pharmaceutical care, most patients reported being satisfied and considered "being well treated" to be the most important aspect during treatment. In addition, all patients (100\%) rated the service as very important and would recommend it to other individuals. Overall, the study showed a high level of patient satisfaction with the service. There is, however, still much to work to be done on this service in order to promote greater access and qualified care to fully achieve a humanized model focused on patient needs.

Key words Chagas disease, Pharmaceutical care, Patient satisfaction
Resumo O Serviço de Atenção Farmacêutica ao paciente com doença de Chagas do estado do Ceará foi criando em 2005, com a finalidade de proporcionar seguimento farmacoterapêutico àqueles com esta morbidade. Decorridos 10 anos de atuação, objetivou-se avaliar a satisfação dos pacientes atendidos no serviço. Tratou-se de um estudo prospectivo, empregando um questionário subdividido nas seções: dados socioeconômicos; infraestrutura, localização e funcionamento; cuidado farmacêutico e importância do serviço. Foram entrevistados 70 pacientes de ambos os sexos e acima de 18 anos, entre agosto de 2014 e maio de 2015. Quanto à infraestrutura, localização e funcionamento, as notas obtidas mostraram que os pacientes estão satisfeitos com os parâmetros analisados. Com relação ao cuidado farmacêutico, a maioria dos pacientes mostrou-se satisfeita, tendo o "ser bem atendido" como aspecto mais importante durante o atendimento. Em relação à importância do serviço, $100 \%$ o considerou muito importante e o indicaria para outras pessoas. De modo geral, o estudo demonstrou um alto nível de satisfação com o serviço. Há muito a ser trabalhado neste serviço, como promover maior acesso e qualificação do atendimento, contribuindo para a concretização de um modelo humanizado, centrado nas necessidades do paciente.

Palavras-chave Doença de Chagas, Atenção farmacêutica, Satisfação do paciente 


\section{Introdução}

A tripanossomíase americana, ou doença de Chagas (DC), é uma infecção sistêmica, de natureza endêmica e de evolução crônica, causada pelo protozoário Trypanosoma cruzi. Estima-se que existam em torno de 6 a 7 milhões de pessoas infectadas em todo o mundo, principalmente na América Latina. Esta doença já esteve confinada somente à América Latina, porém, atualmente, já se espalhou para outros continentes, nas últimas décadas tem sido cada vez mais detectada nos Estados Unidos, Canadá, e muitos países da Europa e alguns países ocidentais do Pacífico ${ }^{1}$.

Dentre as doenças endêmicas, a doença de Chagas ocupa um lugar de destaque, seja pelo grau de morbidade e letalidade, seja pelo alto custo econômico e social que representa. No Brasil, a estimativa é de 2 a 3 milhões de pessoas infectadas, com cerca de 21.000 mortes a cada ano $^{2,3}$.

Na região Nordeste, com exceção do estado da Bahia, Triatoma brasiliensis e Triatoma pseudomaculata são espécies nativas de difícil controle nessa região. A interrupção da transmissão da doença de Chagas por Triatoma infestans teve pouco efeito sobre a transmissão vetorial na maioria dos estados do Nordeste (Maranhão, Ceará, Rio Grande do Norte, Alagoas e Sergipe). Pode-se supor que a mortalidade por Chagas continuará a ser um problema de saúde pública durante décadas nesta região $0^{4,5}$.

O grande desafio dos governantes na década de 80 em relação à doença de Chagas foi desenvolver o programa de controle vetorial, transfusional e melhoria das condições habitacionais ${ }^{6}$. Mesmo após essas medidas de controle, no estado do Ceará foi evidenciado, em estudo entomológico, altos índices de infestação de triatomíneos nos anos de 2009 a $2011^{7}$. Borges-Pereira et al. ${ }^{8}$ encontraram uma prevalência de 3,1\% na população de quatro localidades no município de Jaguaruana-CE, em 2000 a 2002. Em outro estudo, Freitas $^{9}$ encontrou uma soroprevalência de 4,2\% no município de Limoeiro do Norte-CE.

Diante da progressiva redução das atividades de vigilância entomológica, da possibilidade de transmissão ativa da doença e da preocupante realidade da doença de Chagas no estado do Ceará, resta o desafio de prestar assistência aos infectados na fase crônica. Assim, em 2005, foi criado o Serviço de Atenção Farmacêutica aos pacientes com doença de Chagas no estado do Ceará, desenvolvido no Laboratório de Pesquisa em Doen- ça de Chagas (LPDC), situado no Departamento de Análises Clínicas e Toxicológicas da Universidade Federal do Ceará (LPDC/DACT/UFC), na cidade de Fortaleza, com o objetivo de oferecer tratamento e acompanhamento farmacoterapêutico e laboratorial, como também prestar assistência humanizada a esses pacientes.

Além de oferecer um serviço de atenção farmacêutica, o LPDC também funciona como ambiente de ensino, extensão e pesquisa, inserindo os estudantes no ambiente da pesquisa com os pacientes. O Laboratório de Pesquisa em Doença de Chagas desenvolve habilidade de comunicação, competência para informar sobre medicamentos e participa na prevenção e promoção a saúde.

Os pacientes que chegam ao serviço de atenção farmacêutica apresentam dois exames sorológicos positivos de princípios diferentes para a infecção pelo T. cruzi e a prescrição médica de benzonidazol. $\mathrm{O}$ atendimento dos pacientes, cuja sistemática é padronizada, vem sendo realizado por profissionais farmacêuticos e estudantes de graduação e pós-graduação (mestrandos e doutorandos) do curso de Farmácia, previamente treinados.

No serviço, além da dispensação do benzonidazol são realizados: monitorização de parâmetros hematológicos e bioquímicos antes, durante e após o tratamento; monitorização das taxas sorológicas pela técnica de ELISA como critério de cura; como também em cada atendimento são realizadas aferição de pressão, medida da circunferência abdominal, verificação de peso, orientações sobre a farmacoterapia e hábitos de vida saudáveis.

Após 10 anos de implantação do Serviço de Atenção Farmacêutica, propõe-se avaliar a satisfação dos pacientes atendidos. A opinião dos usuários representa apenas uma parte do que é possível investigar sobre os serviços de saúde, porém, esse tipo de pesquisa auxilia gestores e outros profissionais do sistema, possibilitando que a intervenção contemple questões relacionadas a diferenças sociais, educacionais e culturais encontradas e soluções para melhorar a abordagem global do usuário ${ }^{10}$.

Definir a satisfação tem sido uma tarefa difícil para muitos autores, muitos estudos buscaram estudar a relação entre a satisfação e as variáveis que a determinam. Porém, pouco se sabe sobre a natureza ou o número de fatores que influenciam a satisfação $0^{11,12}$. Newsome e Wrigth ${ }^{13}$ mostraram que a satisfação é uma combinação complexa do emocional, físico e imaterial, e seus valores estão 
diretamente envolvidos com a qualidade. Desse modo, exige-se uma constante investigação, na busca de identificar os fatores que promovem satisfação nos pacientes atendidos nos serviços de saúde.

$\mathrm{Na}$ avaliação dos serviços de saúde, devemse considerar vários aspectos da estrutura como recursos físicos, materiais e humanos em relação à quantidade; o processo, que engloba as atividades e procedimentos empregados; resultados, que nos mostra os efeitos das ações e dos procedimentos sobre o estado de saúde do usuário como resultante da assistência recebida; acesso ao serviço; estrutura física e institucional; a relação profissional-paciente; questões financeiras e aspectos relacionados à melhoria e manutenção à saúde ${ }^{14-16}$.

De uma forma geral, existem dois grandes grupos de fatores relacionados com a satisfação: relativo ao serviço e aos provedores e outro inerente aos próprios usuários ${ }^{17}$. Weiss ${ }^{18}$, em seu trabalho sobre os determinantes da satisfação, apresentou quatro grupos principais de determinantes da satisfação que são características dos pacientes, incluindo as sociodemográficas; as expectativas destes sobre a consulta médica e os seus estados de saúde; características dos profissionais que prestam o atendimento, incluindo traços de personalidade, qualidade técnica e a arte do cuidado; aspectos da relação médico-paciente; fatores estruturais e ambientais, incluindo o acesso, forma de pagamento, tempo de tratamento, marcação de consulta e outros.

As pessoas estão exigindo cada vez mais qualidade nos serviços, principalmente dos serviços públicos. Pacientes com menor nível socioeconômico e menor renda tendem a criar menor expectativa, o que pode gerar, portanto, menor censo crítico, avaliando os serviços de forma positiva $^{19-21}$.

No Brasil, o tema satisfação dos usuários com os serviços de saúde vem crescendo em importância especialmente a partir da década de 1990, com o desenvolvimento do Sistema Único de Saúde (SUS) ${ }^{22}$. Apesar disso, a análise da literatura sobre o tema deste estudo, evidencia um numero relativamente baixo de estudos sobre a avaliação da contribuição da atenção farmacêutica a pacientes com doenças negligenciadas, $e$ ao nosso conhecimento, inexistem estudos sobre este tema, bem como, projetos de Atenção Farmacêutica a pacientes portadores de doença de Chagas no Brasil.

Assim, estudos dessa natureza são fundamentais na criação, avaliação e no aprimoramento de serviços de atenção farmacêutica, na busca da humanização e na melhoria contínua do cuidado ao usuário.

\section{Metodologia}

\section{Delineamento, local e população do estudo}

Trata-se de um estudo descritivo prospectivo realizado através da aplicação de questionários aos pacientes com doença de Chagas atendidos no serviço de atenção farmacêutica. Este serviço é desenvolvido no Laboratório de Pesquisa em Doença de Chagas(LPDC), situado no Departamento de Análises Clínicas e Toxicológicas da Universidade Federal do Ceará (LPDC/DACT/ UFC), na cidade de Fortaleza.

Foram incluídos no estudo pacientes de ambos os sexos, na faixa etária acima de 18 anos, com no mínimo seis meses de acompanhamento farmacoterapêutico no serviço de atenção farmacêutica. Foram excluídos do estudo usuários que tinham algum tipo de deficiência que dificulta a fala e/ou a compreensão.

A amostra foi composta de todos os pacientes que compareceram ao serviço no período de agosto de 2014 a maio de 2015, totalizando 70 pacientes, representando $54 \%$ do total de pacientes atendidos neste período. Todos os participantes assinaram o Termo de Consentimento Livre e Esclarecido (TCLE) autorizando a pesquisa.

\section{Aspectos éticos}

O projeto foi aprovado pelo Comitê de Ética em Pesquisa da Universidade Federal do Ceará (COMEPE-UFC) e Certificado de Apresentação para Apreciação Ética (CAAE), estando de acordo com as normas do Conselho Nacional de Saúde (Resolução CNS 466/12).

\section{Coleta de dados}

O questionário foi elaborado baseado no modelo de estudo de Gondim e Andrade ${ }^{23}$. Com esse questionário foi realizado um estudo piloto com 10 pacientes para avaliar a compreensão das questões pelos entrevistados e a factibilidade para execução das entrevistas, após o qual o questionário foi revisto. O questionário possuía 24 questões fechadas distribuídas em 4 partes: dados socioeconômicos (idade, sexo, estado conjugal, escolaridade, renda familiar); infraestrutura, localização e funcionamento (limpeza dos 
espaços, conforto do ambiente, equipamento em funcionamento; silêncio durante o atendimento, privacidade no atendimento, aparência do laboratório, iluminação, sinalização das salas, segurança do local, localização, dia e horário do atendimento); cuidado do farmacêutico (respeito e educação, atenção, interesse, tempo de espera, repasse de informações sobre a doença, tratamento e resultados de exames, discriminação) e importância do serviço (indicação do serviço, aspectos importantes durante o atendimento, como o serviço é considerado). Essa divisão foi baseada nos determinantes da satisfação descritas por Weiss ${ }^{18}$ como as Características dos pacientes, incluindo as características sociodemográficas, em que é possível analisar o perfil social e econômico dos pacientes que pode influenciar no padrão de respostas; Fatores estruturais e ambientais, permitindo-se verificar a adequação do LPDC para a realização dos atendimentos; Características dos profissionais que realizam o atendimento (respeito, educação e atenção com o paciente), sendo um dos principais fatores associados com a satisfação do paciente ${ }^{24}$. O questionário foi aplicado individualmente, por um pesquisador previamente treinado que não fazia parte da equipe do serviço de atenção farmacêutica. No momento em que aguardavam atendimento no LPDC, os pacientes foram abordados e convidados a participar da entrevista, foram esclarecidos pelo entrevistador que o objetivo do estudo era verificar se os usuários estavam satisfeitos com a estrutura física do laboratório e com o atendimento farmacêutico fornecido pelo LPDC. A entrevista foi realizada em uma sala isolada do LPDC para preservar a privacidade do entrevistado. Também foi esclarecido que o estudo era confidencial, sem quaisquer custos ao paciente ou compensações financeiras e que a recusa em participar não traria nenhum prejuízo em sua relação com o pesquisador, com a instituição ou na qualidade do serviço recebido.

\section{Análise dos dados}

Para a análise dos dados foram calculadas frequências para as variáveis categóricas, utilizando um programa Microsoft Office Excel 2007 para fazer as análises estatísticas descritivas e médias para variáveis contínuas. A análise descritiva foi feita por meio da média, desvio padrão, mediana e porcentagem.

A infraestrutura, localização e funcionamento do LPDC foram avaliados através de notas de 0 a 10 atribuídas pelos entrevistados para às variá- veis analisadas (Quadro 1). Foi calculada a média de notas obtidas em cada variável e observada a maior e menor nota. O Cuidado Farmacêutico e Importância do Serviço foram classificados e avaliados de acordo com o Quadro 1.

\section{Resultados}

O presente estudo avaliou um total de 70 pacientes com doença de Chagas atendidos no LPDC no período de agosto de 2014 a maio de 2015, não houve resistência por parte dos pacientes em participar da entrevista. Ao serem explicados sobre o motivo da abordagem, todos aceitaram participar. Quanto às características sociodemográficas, foi observado que $55,70 \%$ dos pacientes eram do sexo feminino. Em relação à ocupação, a maioria relatou ser agricultor $(34,28 \%)$, seguido de aposentado $(21,43 \%)$ e dona de casa $(15,71 \%)$. Os níveis de escolaridade predominantes foram fundamental incompleto $(44,31 \%)$ e analfabeto $(20 \%)$. Quanto à renda familiar, a maioria relatou ganhar menos de um salário mínimo $(67,14 \%)$ ou um salário mínimo (21,43\%) (Tabela 1). A média de idade encontrada dos pacientes foi 52,93 anos.

A maioria dos entrevistados era procedente do interior do Ceará $(88,57 \%)$, com a seguinte distribuição: $27,14 \%$ do município de Limoeiro do Norte; $21,43 \%$ do município de Quixeré; $10 \%$ do município de Russas; $4,26 \%$ do município de Jaguaruana e demais 25,74\% (Tabela 1).

Em relação à infraestrutura, localização e funcionamento do LPDC, a média de notas em todos os parâmetros variou de 9,25 a 9,73 , com isso, percebe-se que a grande maioria dos pacientes deu nota 10 para os parâmetros analisados (Tabela 2).

Alguns pacientes deram notas iguais ou menores que $7 \mathrm{em}$ alguns parâmetros como o conforto do ambiente (4\%); equipamentos em funcionamento (5\%); privacidade no atendimento (4\%); aparência do laboratório (4\%); sinalização das salas (8\%); segurança do local (8\%); localização (7\%); dia do atendimento (5\%) e horário do atendimento $(12 \%)$.

Em relação à satisfação dos pacientes com os cuidados farmacêuticos, os parâmetros analisados (respeito e educação) demonstrados pelo profissional durante o atendimento, a pesquisa mostrou que $69 \%$ dos entrevistados afirmaram estar muito satisfeitos e $31 \%$ responderam estar satisfeitos com atuação da equipe do LPDC. O parâmetro atenção demonstrada pelo farmacêutico em relação à saúde do paciente foi seme- 
Quadro 1. Variáveis estudadas, modo de avaliação e de análises.

\begin{tabular}{|c|c|c|}
\hline \multicolumn{3}{|c|}{ INFRAESTRUTURA, LOCALIZAÇÃO E FUNCIONAMENTO DO LPDC } \\
\hline Variável & Avaliação & Análise da avaliação \\
\hline $\begin{array}{l}\text { - Limpeza dos espaços } \\
\text { - Conforto do ambiente } \\
\text { - Equipamentos em funcionamento } \\
\text { - Silêncio durante atendimento } \\
\text { - Privacidade no atendimento } \\
\text { - Aparência do laboratório } \\
\text { - Iluminação } \\
\text { - Sinalização das salas } \\
\text { - Segurança do local } \\
\text { - Localização } \\
\text { - Dia do atendimento } \\
\text { - Horário do atendimento }\end{array}$ & Nota entre 0 e 10 & $\begin{array}{l}\text { Média das notas } \\
\text { atribuídas a cada } \\
\text { variável }\end{array}$ \\
\hline \multicolumn{3}{|c|}{ CUIDADO FARMACÊUTICO } \\
\hline Variável & Avaliação & Análise da avaliação \\
\hline $\begin{array}{l}\text { - Respeito e educação } \\
\text { - Atenção por parte da equipe do LPDC } \\
\text { - Tempo de espera para receber atendimento } \\
\text { - Interesse pela sua saúde por parte da equipe do LPDC } \\
\text { - Repasse de informações sobre a doença de Chagas } \\
\text { - Repasse de informações sobre o tratamento da doença de } \\
\text { Chagas } \\
\text { - Repasse de informações sobre os resultados dos exames }\end{array}$ & $\begin{array}{l}\text { Muito satisfeito }=1 \\
\text { Satisfeito }=2 \\
\text { Pouco Satisfeito = } 3 \\
\text { Insatisfeito }=4\end{array}$ & $\begin{array}{l}\text { Frequência da } \\
\text { categorização para } \\
\text { cada variável }\end{array}$ \\
\hline - Capacitação dos farmacêuticos e estudantes de farmácia & $\begin{array}{l}\text { Muito preparados }=1 \\
\text { Preparados }=2 \\
\text { Pouco preparados }=3 \\
\text { Não são preparados }=4\end{array}$ & $\begin{array}{l}\text { Frequência da } \\
\text { categorização }\end{array}$ \\
\hline $\begin{array}{l}\text { - Importância dos farmacêuticos e estudantes de farmácia } \\
\text { no atendimento }\end{array}$ & $\begin{array}{l}\text { Ser ouvido pelo } \\
\text { profissional e falar o que } \\
\text { quiser }=1 \\
\text { Sentir-se bem aceito = } 2 \\
\text { Compreender as } \\
\text { orientações dadas = } 3 \\
\text { Sentir competência por } \\
\text { parte do profissional = } 4 \\
\text { Ser bem acolhido pelo } \\
\text { profissional = 5 }\end{array}$ & $\begin{array}{l}\text { Frequência da } \\
\text { categorização }\end{array}$ \\
\hline \multicolumn{3}{|c|}{ IMPORTÂNCIA DO SERVIÇO } \\
\hline Variável & Avaliação & Análise da avaliação \\
\hline - Indicação do serviço & $\begin{array}{l}\text { Sim }=1 \\
\text { Não }=2 \\
\text { Não sei }=3\end{array}$ & $\begin{array}{l}\text { Frequência da } \\
\text { categorização }\end{array}$ \\
\hline - Relevância do serviço realizado pelo LPDC & $\begin{array}{l}\text { Muito importante }=1 \\
\text { Pouco importante }=2 \\
\text { Não é importante }=3\end{array}$ & $\begin{array}{l}\text { Frequência da } \\
\text { categorização }\end{array}$ \\
\hline
\end{tabular}

Fonte: Autoria própria.

lhante aos resultados acima, 70\% referiram estar muito satisfeitos e 30\% satisfeitos (Tabela 3).

Ao serem perguntados sobre a competência dos estudantes de farmácia e farmacêuticos do serviço (transmitir as informações necessárias; realizar os procedimentos adequados; esclarecer dúvidas), 39\% dos pacientes responderam que estes estavam muito preparados para as atividades desenvolvidas e $61 \%$ responderam que estavam preparados. Observou-se ainda que, 63\% 
Tabela 1. Características sociodemográficas dos pacientes com doença de Chagas atendidos no serviço de atenção farmacêutica, no período agosto de 2014 a maio de $2015(\mathrm{n}=70)$.

\begin{tabular}{|c|c|c|c|}
\hline Variáveis & Categoria & $\mathbf{N}$ & $\%$ \\
\hline \multirow[t]{2}{*}{ Sexo } & Feminino & 39 & 55,70 \\
\hline & Masculino & 31 & 44,30 \\
\hline \multirow[t]{2}{*}{ Idade } & $18-59$ anos & 52 & 74,30 \\
\hline & $\geq 60$ anos & 18 & 25,70 \\
\hline \multirow[t]{7}{*}{ Ocupação } & Agricultor & 24 & 34,28 \\
\hline & Aposentado & 15 & 21,43 \\
\hline & Dona de Casa & 11 & 15,71 \\
\hline & Desempregado & 03 & 4,26 \\
\hline & Professor & 03 & 4,26 \\
\hline & Motorista & 03 & 4,26 \\
\hline & Outros & 11 & 15,71 \\
\hline \multirow[t]{6}{*}{ Escolaridade } & Analfabeto & 14 & 20,00 \\
\hline & Fundamental incompleto & 31 & 44,31 \\
\hline & Fundamental completo & 11 & 15,71 \\
\hline & Médio incompleto & 03 & 4,26 \\
\hline & Médio completo & 09 & 12,86 \\
\hline & Superior & 02 & 2,86 \\
\hline \multirow[t]{7}{*}{ Procedência } & Estado do Ceará & 68 & 97,14 \\
\hline & Limoeiro do Norte & 19 & 27,14 \\
\hline & Quixeré & 15 & 21,43 \\
\hline & Russas & 07 & 10,00 \\
\hline & Outros municípios & 27 & 38,57 \\
\hline & Estado do Maranhão & 01 & 1,43 \\
\hline & Estado do Rio Grande do Norte & 01 & 1,43 \\
\hline \multirow[t]{3}{*}{ Renda Familiar } & $<1 \mathrm{SM}$ & 47 & 67,14 \\
\hline & $1 \mathrm{SM}$ & 15 & 21,43 \\
\hline & 2 a 4 SM & 08 & 11,43 \\
\hline
\end{tabular}

Fonte: Autoria própria. $\mathrm{SM}=$ Salário mínimo.

Tabela 2. Média de notas obtidas dos pacientes com doença de Chagas atendidos no serviço de atenção farmacêutica em relação aos parâmetros analisados (infraestrutura, localização e funcionamento), no período agosto de 2014 a maio de $2015(\mathrm{n}=70)$.

\begin{tabular}{llcc}
\hline \multicolumn{1}{c}{ Variáveis } & Média de notas & Menor nota & Maior nota \\
\hline Limpeza dos espaços & 9,614 & 7,000 & 10,000 \\
Conforto do ambiente & 9,314 & 7,000 & 10,000 \\
Equipamentos em funcionamento & 9,485 & 5,000 & 10,000 \\
Silêncio durante atendimento & 9,728 & 5,000 & 10,000 \\
Privacidade no atendimento & 9,657 & 6,000 & 10,000 \\
Aparência do laboratório & 9,414 & 6,000 & 10,000 \\
Iluminação & 9,471 & 5,000 & 10,000 \\
Sinalização das salas & 9,371 & 0,000 & 10,000 \\
Segurança do local & 9,257 & 3,000 & 10,000 \\
Localização & 9,385 & 5,000 & 10,000 \\
Dia do atendimento & 9,542 & 0,000 & 10,000 \\
Horário do atendimento & 9,371 & 5,000 & 10,000 \\
\hline
\end{tabular}

Fonte: Autoria própria. 
Tabela 3. Satisfação dos pacientes com doença de Chagas atendidos no serviço de atenção farmacêutica em relação aos cuidados farmacêuticos, no período agosto de 2014 a maio de $2015(\mathrm{n}=70)$.

\begin{tabular}{|c|c|c|}
\hline & $\mathrm{N}$ & $\%$ \\
\hline \multicolumn{3}{|c|}{ Respeito e educação durante o atendimento } \\
\hline Muito Satisfeito & 48 & 68,57 \\
\hline Satisfeito & 22 & 31,43 \\
\hline Pouco Satisfeito & - & - \\
\hline Insatisfeito & - & - \\
\hline \multicolumn{3}{|c|}{ Atenção durante o atendimento } \\
\hline Muito Satisfeito & 49 & 70,0 \\
\hline Satisfeito & 21 & 30,0 \\
\hline Pouco Satisfeito & - & - \\
\hline Insatisfeito & - & - \\
\hline \multicolumn{3}{|l|}{ Tempo de espera } \\
\hline Muito Satisfeito & 29 & 41,43 \\
\hline Satisfeito & 38 & 54,28 \\
\hline Pouco Satisfeito & 3 & 4,29 \\
\hline Insatisfeito & - & - \\
\hline \multicolumn{3}{|c|}{ Interesse pela sua saúde } \\
\hline Muito Satisfeito & 44 & 62,85 \\
\hline Satisfeito & 26 & 37,15 \\
\hline Pouco Satisfeito & - & - \\
\hline Insatisfeito & - & - \\
\hline \multicolumn{3}{|c|}{ Informações sobre a doença de Chagas } \\
\hline Muito Satisfeito & 46 & 65,71 \\
\hline Satisfeito & 23 & 32,86 \\
\hline Pouco Satisfeito & 1 & 1,43 \\
\hline Insatisfeito & - & - \\
\hline \multicolumn{3}{|c|}{ Informações sobre os exames realizados } \\
\hline Muito Satisfeito & 20 & 28,58 \\
\hline Satisfeito & 33 & 47,14 \\
\hline Pouco Satisfeito & 16 & 22,85 \\
\hline Insatisfeito & 1 & 1,43 \\
\hline \multicolumn{3}{|c|}{ Informações sobre o tratamento } \\
\hline Muito Satisfeito & 44 & 62,85 \\
\hline Satisfeito & 26 & 37,15 \\
\hline Pouco Satisfeito & - & - \\
\hline Insatisfeito & - & - \\
\hline \multicolumn{3}{|c|}{ Farmacêuticos e Estudantes } \\
\hline Muito preparado & 27 & 38,60 \\
\hline Preparado & 43 & 61,40 \\
\hline Pouco preparado & - & - \\
\hline Não preparados & - & - \\
\hline \multicolumn{3}{|l|}{ Discriminação } \\
\hline Sim & - & \\
\hline Não & 70 & 100 \\
\hline
\end{tabular}

Fonte: Autoria própria.

dos pacientes estavam muito satisfeitos e, 37\%, satisfeitos com o interesse que os profissionais demonstravam pela sua saúde (consequências da doença, outras comorbidades).
Com relação ao repasse de informações sobre a doença de Chagas (como agente causador da doença, formas de transmissão, formas clínicas, diagnóstico), $66 \%$ dos pacientes afirmaram estar muito satisfeitos, 33\% satisfeitos e, apenas $1 \%$, pouco satisfeitos. E em relação ao repasse de informações sobre o tratamento da doença de Chagas (benefícios e riscos do medicamento Benzonidazol), 63\% dos pacientes responderam estar muito satisfeitos e $37 \%$ satisfeito.

Quando questionados sobre o repasse de informações sobre os exames (acompanhamento sorológico), os resultados indicam que $29 \%$ estavam muito satisfeitos; $47 \%$ satisfeitos; porém, observou-se que $23 \%$ estavam pouco satisfeitos e $1 \%$ estavam insatisfeitos.

Solicitou-se ainda aos pacientes que escolhessem, entre as opções sugeridas, o que eles consideravam mais importante no atendimento realizado no serviço, podendo optar por mais de uma resposta. Observou-se que $37,18 \%$ consideraram o bom acolhimento pelo profissional como o ponto mais importante no atendimento, $26,9 \%$ ser ouvido pelo profissional e poder falar o que quiser; $14,10 \%$ sentir-se bem aceito; $12,82 \%$ compreender as orientações dadas (Tabela 4). Quando questionados quanto à indicação do serviço para outros pacientes, todos disseram que indicariam e classificaram o serviço como muito importante.

\section{Discussão}

As doenças crônicas têm um grande impacto na morbidade e mortalidade de pacientes e representam um desafio para os serviços de saúde. Pacientes com doenças crônicas podem se beneficiar de estratégias preventivas proporcionadas pela atenção farmacêutica para detectar e tratar complicações, numa fase inicial, reduzindo a morbidade e mortalidade e, consequentemente, os custos relacionados com a saúde, mostrando a importância de serviços farmacêuticos, que atualmente ainda são $\operatorname{pou}^{2} \cos ^{25}$, o que dificulta a comparação dos resultados deste estudo com outros.

No presente estudo observou-se um alto grau de satisfação com o serviço, porém, este dado deve ser muito bem avaliado devido a três fatores principais: a característica da população, o medo de perder o serviço e a satisfação com o acolhimento que pode influenciar na avaliação dos outros itens que mereceriam notas menores.

O perfil dos usuários atendidos no serviço de atenção farmacêutica é do sexo feminino acima 
Tabela 4. Importância do serviço de atenção farmacêutica ao paciente com doença de Chagas, no período agosto de 2014 a maio de $2015(\mathrm{n}=70)$.

\begin{tabular}{llr}
\hline \multicolumn{1}{c}{ Variáveis } & $\mathbf{n}$ & $\%$ \\
\hline Indicação do serviço & 70 & 100 \\
$\quad$ Sim & - & - \\
Não & & \\
Serviço realizado no LPDC & 70 & 100 \\
$\quad$ Muito importante & - & - \\
$\quad$ Pouco importante & - & - \\
Não importante & & \\
Aspecto importante no atendimento & \\
$\quad$ Ser ouvido pelo profissional e falar o que quiser & 21 & 26,9 \\
Sentir-se bem aceito & 11 & 14,10 \\
Compreender as orientações dadas & 10 & 12,82 \\
Sentir competência por parte do profissional & 07 & 9,0 \\
Ser bem acolhido pelo profissional & 29 & 37,18 \\
\hline
\end{tabular}

Fonte: Autoria própria.

${ }^{*}$ O paciente pode escolher mais de uma opção.

de 50 anos, agricultor ou aposentado, de baixo nível de escolaridade, ganhando menos de um salário mínimo, procedentes da zona rural do Ceará. Logo se trata de usuários de baixa escolaridade e de situação socioeconômica precária. Estudos mostram que estes tendem a ser menos rigorosos na avaliação dos serviços que lhes são prestados, pois a falta de recursos financeiros dessa faixa da população não lhe dá alternativas. Dessa forma, qualquer oportunidade que apareça em um momento de necessidade acaba sendo recebida com grande satisfação, mesmo que a qualidade do serviço não seja a ideal. As pessoas com nível de educação mais avançado desenvolvem uma consciência crítica e exigem serviços de melhor qualidade para satisfazer suas expectativas $^{19-21}$.

O serviço de atenção farmacêutica se localiza na área do complexo hospitalar, próximo ao Centro de Hematologia e Hemoterapia do Ceará (HEMOCE), e a grande maioria dos pacientes é procedente do interior do Ceará (Tabela 1). Os pacientes se deslocam para o laboratório através de transportes disponibilizados pela Secretaria de Saúde de cada município, mesmo com a distância os pacientes se mostram satisfeitos com a localização do serviço (Tabela 2). Estudos discutem que a alta satisfação dos pacientes nos serviços de saúde, apesar de todas as limitações observadas, pode expressar o receio dos usuários em perder o direito ao serviço, fazendo que eles avaliem sempre positivamente ${ }^{26}$.
Outro fator a considerar seria o fato de tratarse de uma doença negligenciada e este serviço ser exclusivo para pacientes portadores de doença de Chagas. Dessa forma os pacientes acabam avaliando positivamente o serviço, pois não existem outros serviços farmacêuticos equivalentes no Ceará que eles possam comparar ou que sirvam de referência. Muitos pacientes não têm conhecimento pleno dos seus direitos e deveres, o que pode reduzir a capacidade crítica dos pacientes para avaliar com mais objetividade suas percepções quanto ao serviço que lhes é prestado ${ }^{19-21}$.

Moimaz et al. ${ }^{24}$, em seu estudo sobre a Satisfação e percepção do usuário do SUS sobre o serviço público de saúde, verificaram que os recursos estavam sucateados e em desuso. Mesmo assim, a satisfação do paciente foi elevada. Diante disso, observaram a relevância da qualidade do atendimento aos usuários como constructo determinante do seu nível de satisfação, destacando que aspectos tais como agilidade, responsividade e cortesia são importantes para os usuários do serviço de saúde.

Novaes $^{27}$ enfatizou que a estrutura física do serviço de saúde é um fator importante na qualidade do serviço prestado, porém não é o único fator para garantir o sucesso dos serviços. Para isso, é necessária uma ligação entre a equipe multidisciplinar com as condições de infraestrutura. No presente estudo, um pequeno percentual dos participantes não aprovou os parâmetros de infraestrutura, que apesar de não representar significância, indicam que intervenções pontuais devam ser realizadas para atender as necessidades do usuário e proporcionar assim um melhor atendimento. É fato que o LPDC dispõe de um pequeno espaço para atender os pacientes do serviço de atenção farmacêutica. Diante da proporção que esse serviço tomou, necessita-se de um espaço maior, mais confortável e melhor equipado para o atendimento e para a realização de exames dos pacientes, proporcionando assim um atendimento de qualidade.

Muitos estudos mostram que, na atenção em saúde, o cuidado humanizado implica também na qualidade do serviço, sendo um indicador muito importante para a satisfação dos pacientes $^{28,29}$. Diante disso, observa-se que os pacientes estão satisfeitos com o respeito e educação recebidos por parte da equipe do LPDC. Samico et al. ${ }^{30}$ mostraram em seu trabalho que o cuidado humanizado e a satisfação dos usuários estão vinculados ao acolhimento dos funcionários e dos profissionais de saúde, em que, na perspectiva do usuário, qualidade da atenção, respeito 
e educação demonstrados pelos funcionários e profissionais são indicadores de satisfação. Gonçalves et al. ${ }^{31}$ avaliaram a satisfação dos pacientes submetidos à intervenção fisioterapêutica, no município de Campo Maior -Piauí. Lá, evidenciaram a alta satisfação dos pacientes com o respeito, privacidade, ótimo relacionamento com os profissionais, além de conforto, boas instalações, limpeza e a satisfação de suas necessidades. Esses parâmetros devem ser abordados constantemente para se avaliar os serviços de saúde.

O LPDC proporciona aos estudantes de farmácia um aprendizado na prática da atenção farmacêutica, onde o discente tem contato com o paciente, atuando diretamente na farmacoterapia. Os graduandos têm a oportunidade de participar e acompanhar as coletas de sangue, também orientam os pacientes quanto à doença em seus aspectos biológicos e patológicos e sobre hábitos saudáveis. Os estudantes esclarecem dúvidas acerca do plano terapêutico; fornecem informações sobre o potencial das complicações; observam as prescrições medicamentosas quanto à administração, horário e dosagens, explicando tudo de forma clara aos pacientes. Além disso, realizam exames sorológicos para o acompanhamento desses pacientes; verificam a pressão arterial dos mesmos e fazem intervenções quando necessárias. Logo, diante dos resultados encontrados, observa-se que os pacientes estão muito satisfeitos com o desempenho dos estudantes de farmácia durante os atendimentos.

Moimaz et al. ${ }^{24}$ e Lopes e Silva ${ }^{32}$ consideraram que o desempenho profissional é avaliado pelo usuário, através do interesse demonstrado no exame físico, nas perguntas feitas e orientações dadas, bem como na resolutividade das condutas. O bom atendimento, baseado na escuta do usuário, e o bom desempenho do profissional propiciam o vínculo entre usuário e o serviço de saúde. Esse vínculo aperfeiçoa o processo da assistência, permitindo que os profissionais conheçam seus pacientes e as prioridades de cada um, facilitando-lhes o acesso ao serviço. É essa relação de respeito, compreensão e escuta que faz a diferença entre as práticas das ações de saúde ${ }^{29,30}$.

Estudos mostram que os pacientes estão satisfeitos com o papel do farmacêutico na área da saúde. Alturki e Khan ${ }^{33}$ investigaram a satisfação do paciente com o farmacêutico, em um hospital da Arábia Saudita, e verificaram que 65,9\%(434) dos entrevistados mencionaram que o farmacêutico tem grande papel na recuperação da saúde. Em outro estudo, Monteiro et al. ${ }^{34}$ verificaram em um serviço de atenção farmacêutica, de um hospital da Espanha, que 56\% dos indivíduos consideram as informações fornecidas pelo farmacêutico muito úteis, auxiliando no conhecimento de seu estado de saúde e no uso correto de medicamentos, e $81,1 \%$ consideram o farmacêutico simpático e atencioso.

Carrascosa et al. ${ }^{35}$ avaliaram a satisfação dos pacientes com um serviço de atenção farmacêutica para pacientes que realizavam tratamentos de fertilidade, em um hospital da Europa, e observaram que o farmacêutico melhorou a adesão dos pacientes ao tratamento. Os pacientes que completaram o estudo estavam 100\% satisfeitos com as informações fornecidas pelo farmacêutico e com o atendimento recebido. O farmacêutico recebeu 9,09 pontos em uma escala de 10, mostrando um alto nível de satisfação do usuário, e concluíram que a atenção farmacêutica é uma importante estratégia para alcançar a adesão ao tratamento pelo paciente.

Durante os atendimentos no LPDC os pacientes recebem informações sobre a doença de Chagas (agente causador, formas de transmissão, formas clínicas e diagnósticas), para que eles tenham conhecimento de sua doença e consciência do seu estado de saúde e cuidados a serem aplicados; os pacientes mostraram-se satisfeitos com o repasse de informações sobre a doença (Tabela 3). Os pacientes também se mostraram satisfeitos com o repasse de informações sobre o tratamento da doença. É essencial que os mesmos estejam cientes sobre os benefícios do tratamento como também sobre os riscos de reações adversas que benzonidazol pode vir a causar, auxiliando assim em uma melhor adesão do tratamento ${ }^{36}$.

Quando questionados sobre o repasse de informações sobre os resultados dos exames realizados no LPDC, verificou-se que os pacientes estavam satisfeitos, porém 22,85\% relataram estarem pouco satisfeitos. Esse resultado deve funcionar como alerta para o serviço, pois essas informações são essenciais para o paciente, para que tenha consciência de seu estado de saúde. Essa fragilidade pode ser justificada pelo fato do LPDC dispor de pouco financiamento, apoio e parcerias com outras instituições, para suprir as deficiências de kits para a realização do acompanhamento sorológico, o que acarreta demora na entrega dos resultados dos exames. Atualmente, 2016, o serviço conta com a parceria do Laboratório Central do Estado (LACEN), que disponibiliza os kits para a realização dos exames e dessa forma possa ser corrigida essa falha do serviço.

Cada vez mais os usuários dos serviços de saúde buscam a resolução de suas necessidades, 
assim como por informações sobre as doenças que os agridem, sobre tratamentos disponíveis, exames realizados. Buscam também a orientação do profissional de saúde que deve possuir conhecimento necessário para tais esclarecimentos, pois tais orientações vão auxiliar na manutenção do bem-estar do indivíduo como também lhes permitirão maior autonomia em relação à própria saúde ${ }^{37}$.

O pequeno espaço para o atendimento farmacêutico no LPDC pode gerar um tempo maior de espera por parte dos pacientes, mesmo com essa fragilidade mostraram-se satisfeitos com o tempo de espera para receber o atendimento. Uma melhor infraestrutura e um maior número de estudantes e profissionais propiciariam um atendimento mais rápido. Em muitos estudos, o tempo de espera para receber atendimento é uma das principais reclamações dos usuários e essa insatisfação tem-se mostrado rotineira nos serviços ${ }^{38,39}$, fato este não observado no presente estudo.

Moimaz et al. ${ }^{24}$, em seu trabalho sobre satisfação dos usuários do SUS, apresentaram resultados que corroboram com o presente estudo, pois verificaram alta satisfação dos pacientes com o cuidado humanizado por parte dos profissionais. $69,5 \%$ dos pacientes relataram estar satisfeitos com todos os profissionais de saúde da rede pública pelos quais foram atendidos, mais da metade $(63,7 \%)$ estavam satisfeitos com o repasse de informações sobre prevenção, $72,9 \%$ satisfeitos com o repasse de informações sobre a doença que tinham e 70,3\% satisfeitos com as informações recebidas sobre os procedimentos que foram realizados para o tratamento. $83,1 \%$ relataram estar satisfeitos com as explicações fornecidas pelos profissionais a respeito da administração dos medicamentos.

Todos os pacientes entrevistados consideram o serviço de atenção farmacêutica muito importante e o indicariam para outras pessoas, demonstrando que na perspectiva dos pacientes, apesar das limitações do LPDC, este tem conseguido fazer um serviço de qualidade.

A alta satisfação a um serviço de atenção farmacêutica pode também ser verificada em outros estudos na literatura, como o de Márquez-Peiró e Pérez-Peiró ${ }^{40}$, que avaliaram a satisfação dos pacientes com os cuidados farmacêuticos em um Hospital Universitário da Espanha. Este retorno do usuário é útil para estabelecer melhorias, como aumento dos recursos estruturais e humanos, buscando sempre um melhor atendimento aos pacientes.

No atendimento farmacêutico, o paciente considerou mais importante ser bem acolhido; ser ouvido pelo profissional e poder falar o que quiser. Dessa forma, o acolhimento, a atenção e o cuidado são fatores de maior relevância para os pacientes, diferentemente dos problemas de infraestrutura e escassez de recursos que existem na maioria dos serviços de saúde. O cuidado humanizado acaba sendo um dos aspectos mais importantes na avaliação da satisfação dos usuários dos serviços de saúde ${ }^{28,41}$.

Por fim, pesquisa de satisfação do usuário retrata resultados temporários e que precisam sempre estar em evidência, pois refletem as condições dos serviços que são oferecidos aos usuários, necessitando de contínuo aprimoramento até mesmo em razão do aumento do número de pacientes $^{10}$.

\section{Conclusão}

Pode-se inferir que os pacientes do LPDC se apresentaram satisfeitos com o cuidado farmacêutico que receberam durante os atendimentos, com as condições de infraestrutura, mesmo que não sejam ideais, não foi uma falha apontada pelos mesmos. Porém, estes dados devem ser interpretados de forma cautelosa devido as características da população estudada, que, como discutido, influencia no padrão de respostas. Sabe-se que ainda há muito a ser trabalhado neste serviço, e, por isso, almeja-se a construção e implementação de uma unidade de atenção farmacêutica para promover maior acesso e qualificação do atendimento, contribuindo para a concretização de um modelo humanizado e centrado nas necessidades do paciente. 


\section{Colaboradores}

AC Costa trabalhou na pesquisa, metodologia, discussão dos resultados, conclusões, bibliografia; DS Cândido no resumo, abstract, metodologia, resultados; ASOB Vasconcelos trabalhou na pesquisa e no instrumento da coleta de dados; JD Silva Filho trabalhou na análise dos resultados e elaboração de tabelas; CEM Viana trabalhou na aplicação do instrumento para a coleta de dados; MC Andrade trabalhou na pesquisa e análise dos resultados; MA Lima trabalhou na metodologia e coleta de dados; MF Oliveira trabalhou na pesquisa, metodologia e discussão dos resultados.

\section{Agradecimentos}

A Fundação Cearense de Apoio ao Desenvolvimento Científico e Tecnológico - FUNCAP pelo apoio recebido.

\section{Referências}

1. World Health Organization (WHO). Chagas disease (American trypanosomiasis). Geneva: WHO; 2016. [cited 2016 Jun 2]. Available from: http://www.who.int/ mediacentre/factsheets/fs340/en/

2. Dias JCP. Globalização, iniquidade e Doença de Chagas. Cad Saude Publica 2007; 23(Supl. 1):513-522.

3. World Health Organization (WHO). Consultation on International Biological Reference. Preparations for Chagas diagnostic tests. Geneva: WHO; 2007. [cited 2016 Jun 19]. Available from: http://www.who.int/ bloodproducts/ref_materials/WHO_Report_1st_Chagas_BRP_consultation_7-2007_final.pdf

4. Braz SCM, Melo MFAD, Lorena VMB, Souza WV, Gomes YM. Chagas disease in the State of Pernambuco, Brazil: analysis of admissions and mortality time series. Rev Soc Bras Med Trop 2011; 44(3):318-323.

5. Martins-Melo FR, Ramos Júnior AN, Alencar $\mathrm{CH}$, Heukelbach J. Mortality related to Chagas disease and HIV/AIDS coinfection in Brazil. J Trop Med 2012; 2012:534649.

6. Schmunis GA. Trypanossomiase americana: seu impacto nas Américas e perspectivas de eliminação. In: Dias JCP, Coura JR, organizadores. Clínica e Terapêutica da Doença de Chagas: uma Abordagem Prática para o Clínico Geral. Rio de Janeiro: Fundação Oswaldo Cruz; 1997. p. 11-23.

7. Vasconcelos Fidalgo ASOB. Índice de infestação e infecção de triatomíneos por Trypanosoma cruzi na região sudeste do Estado do Ceará [dissertação]. Fortaleza: Universidade Federal do Ceará; 2013.

8. Borges-Pereira J, Sarquis O, Zauza PL, Britto C, Lima MM. Epidemiologia da doença de Chagas em quatro localidades rurais de Jaguaruana, Estado do Ceará. Soroprevalência da infecção, parasitemia e aspectos clínicos. Rev. Soc. Bras. Med. Trop 2008; 41(4):345-351.

9. Freitas EC. Inquérito soroepidemiológico da doença de Chagas no município de Limoeiro do Norte, Ceará em 2013 [dissertação]. Fortaleza: Universidade Federal do Ceará; 2014.

10. Chindia ED. Estudo exploratório sobre satisfação do usuário de consultas externas do Hospital Josina Machel Luanda, Angola [dissertação]. Rio de Janeiro: Escola Nacional de Saúde Pública Sérgio Arouca; 2012.

11. Lewis JR. Patient views on quality care in general practice: literature review. Soc Sci Med 1994; 39(5):655-670.

12. Ross CK, Steward CA, Sinacope JM. The importance of patient preferences in the measurement of health care satisfaction. Med Care 1993; 31:1138-1149.

13. Newsome PRH, Wright GH. A review of patient satisfaction: 1 concepts of satisfaction. Br Dent J 1999; 186(4):161-165.

14. Donabedian A. La calidad de laatención médica - definición y métodos de evaluación. Salud Pública de México 1984; 32(2):248-249.

15. Donabedian A. The seven pillars of quality. Arch Pathol Lab Med. 1990; 114(11):1115-1118.

16. Vaistman J, Andrade GRB. Satisfação e responsividade: formas de medir a qualidade e a humanização da assistência à saúde. Cienc Saude Colet 2005; 10(3):599-613.

17. Fox JG, Storms DM. A different approach to social demographic predictors for satisfaction with health care. Soc Sci Med 1981; 15(5):557-567. 
18. Weiss GL. Patient satisfaction with primary medical care: evaluation of sociodemographic and predispositional factor. Med Care 1988; 26(4):383-392.

19. Oliveira DF, Arieta CE, Temporini ER, Kara JN. Quality of health care: patient satisfaction in a university hospital. Arq Bras Oftalmol 2006; 65(5):731-736.

20. Souza A, Griebeler D, Godoy L. Qualidade na prestação de serviços fisioterápicos: estudo de caso sobre expectativas e percepções de clientes. Produção 2007; 17(3):435-453.

21. Machado NP, Nogueira, LT. Avaliação da satisfação dos usuários de serviços de Fisioterapia. Rev Bras Fisioter 2008; 12(5):401-408.

22. Gouveia GC, Souza WV, Luna CF, Souza-Júnior PRB, Szwarcwald CL. Health care users' satisfaction in Brazil. Cad Saude Publica 2005; 21(Supl. 1):S109-S118.

23. Gondim APS, Andrade JT. Cuidado humanizado na atenção primária à saúde: demanda por serviços e atuação profissional na rede de atenção primária á saúde - Fortaleza, Ceará, Brasil. Rev Port Saúde Pública 2014; 32(1):61-68.

24. Moimaz SAS, Marques JAM, Saliba O, Garbin CAS, Zina LG, Saliba NA. Satisfação e percepção do usuário do SUS sobre o serviço público de saúde. Revista de Saúde Coletiva 2010; 20(4):1419-1440.

25. Brasil. Ministério as Saúde (MS). Plano de ações estratégicas para o enfrentamento das Doenças Crônicas Não transmissiveis (DCNT) no Brasil 2011-2022. Brasília: MS; 2011.

26. Gomes M, Barbosa EF. A técnica de grupos focais para obtenção de dados qualitativos. Revista Educativa 1999.

27. Novaes HMD. Pesquisa em, sobre e para os serviços de saúde: panorama internacional e questões para a pesquisa em saúde no Brasil. Cad Saude Publica 2004; 20(2):147-173.

28. Schuster MA, Mcglynn EA, Brook RH. How good is the quality of health care in the United States? Milbank $Q$ 2005; 83(4):843-895.

29. Choudry NK, Fletcher RH, Soumerai SB. Systematic review: The relationship between clinical experience and quality of health care. Ann Intern Med 2005; $142(4): 260-273$.

30. Samico I, Hartz ZMA, Felisberto E, Carvalho EF. Atenção à saúde da criança: uma análise do grau de implantação e da satisfação de profissionais e usuários em dois municípios do estado de Pernambuco. Rev. Bras. Sáude Mater Infant. 2005; 5(2):229-240.

31. Gonçalves JR, Veras FEL, Matos ACM, Lima ISA. Avaliação da satisfação dos pacientes submetidos à intervenção fisioterapêutica no município de Campo Maior, PI. Fisioter Mov 2011; 24(1):47-56.

32. Lopes MJM, Silva JLA. Estratégias metodológicas de educação e assistência na atenção básica de saúde. Rev Latino am Enfermagem 2004; 12(4):683-688.
33. Alturki M, Khan TM. A study investigating the level of satisfaction with the health services provided by the Pharmacist at ENT hospital, Eastern Region Alahsah, Kingdom of Saudi Arabia. Saudi Pharmaceutical Journal 2013; 21:255-260.

34. Montero A, Feal B, Calvín M, Margusino L, Hurtado JL, Vázquez I, Martínez A, Jorge S, Martín I. Calidad percibida por los pacientes externos em la consulta de atención farmacêutica del servicio de farmácia. Farmacia Hospitalaria 2006; 30(2):105-111.

35. Carrascosa FS, Cantero MD, Fernández MJH, Hernández IR, Muñoz CG, Martín FG, Sánchez GB, López MD, Olid AS, Martín MVM. DI-048 Pharmaceutical care programme in fertility treatments: Analysis of patient satisfaction levels. Eur J Hosp Pharm 2016; 23(1):38-39.

36. Beney J, Bero LA, Bond C. Expanding the roles of outpatient pharmacists: effects on health services utilization, costs, and patient outcomes. Cochrane Database Syst Rev 2000; (3):CD000336.

37. Lima MADS, Ramos DD, Rosa RB, Nauderer TM, Davis $\mathrm{R}$. Acesso e acolhimento em unidades de saúde na visão dos usuários. Acta Paul Enferm 2007; 20(1):12-17.

38. Andrade KLC, Ferreira EF. Avaliação da inserção da odontologia no Programa Saúde da Família de Pompeu (MG):a satisfação do usuário. Cien Saude Colet 2006; 11(1):123-130.

39. Franco SC, Campos GWS. Avaliação da qualidade de atendimento ambulatorial em pediatria em um hospital universitário. Cad Saude Publica 1998; 14(1):61-70.

40. Márquez-Peiró JF, Pérez-Peiró C. Evaluación de la satisfacción y de la insatisfacción de pacientes atendidos en la unidad de atención farmacéutica a pacientes externos. Farmacia Hospitalaria 2008; 32(2):71-76.

41. Medeiros FA, Souza GCA, Barbosa AAA, Costa ICC. Acolhimento em uma Unidade Básica de Saúde: a satisfação do usuário em foco. Rev. salud pública 2010; 12(3):402-413.

Artigo apresentado em 30/10/2015

Aprovado em 20/07/2016

Versão final apresentada em 22/07/2016 